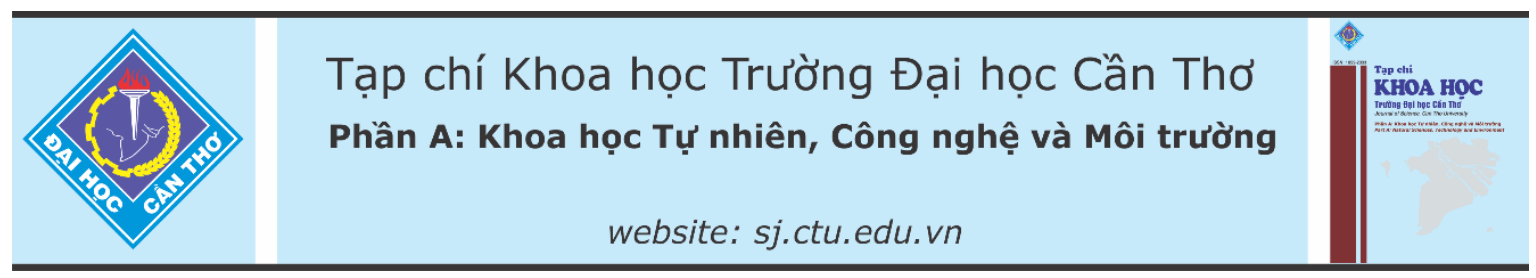

DOI:10.22144/ctu.jvn.2021.031

\title{
THIẾT KẾ ROBOT ĐIỀU KHIỂN CAMERA DI CHUYỂN TRÊN RAY CHO TRƯỜNG QUAY ĐÀI TRUYỀN HİNH
}

\author{
Nguyễn Hoàng Vương ${ }^{1}$, Nguyễn Chí Ngôn ${ }^{2}$ và Hồ Hữu Trung ${ }^{2}$ \\ ${ }^{1}$ Trung tâm Truyền hình Việt Nam Khu vưc Nam Bộ \\ ${ }^{2}$ Khoa Công nghệ, Truờng Đại học Cần Tho \\ *Ngưòi chịu trách nhiệm về bài viết: Nguyễn Hoàng Vuơng (email: nhvuongcvtv@gmail.com)
}

\section{Thông tin chung:}

Ngày nhận bài: 23/10/2020

Ngày nhận bài sưa: 06/11/2020

Ngày duyệt đăng: 28/04/2021

\section{Title:}

Design of camera control robot moving on ray for television studio

\section{Tù khóa:}

Điều khiển camera, điều khiển $P I D$, điều khiển $M \grave{o}-P I D$, điều khiến pan-tilt, hệ thống dolly

\section{Keywords:}

Camera control, pid control, fuzzy-pid control, pan-tilt control, dolly system

\begin{abstract}
Since its inception until now, TV communication has been an indispensable part of life. The quality of life is increasingly improved, the entertainments for life also comes along. In addition to increasing image quality, the image reflected on the conveyed content is also an important part. That is the device that supports the camera to help create flexible, sweeping image angles, without real shaking the content passed to the viewer. That supporting device mentioned in this paper is the camera control system for moving on ray. This system was previously adopted as a rudimentary system with two manual operators and took up a lot of space within the studio and was very passive. It is improved with intelligent control system to help the camera move as desired and especially stable, the image does not shake when the camera is pan, tilt and move. The automatic control function for stable system operation is applied by a proportional integral derivative (PID) controller. The simulation results on MATLAB ${ }^{\circledR}$ are presented and the test results on the actual model for this system meet the set goals.
\end{abstract}

\section{TÓM TẮT}

Tù khi mới hình thành cho đến nay, truyền thông truyền hình, là một phần không thể thiếu trong cuộc sống. Chất lượng cuộc sống ngày càng được nâng cao thi giải trí phục vu cuộc sống cũng song song đi kèm. Ngoài chất luợng hình ảnh có độ nét ngày càng cao, hình ảnh phản ảnh lên nội dung chuyển tải cung là một phần quan trọng. Đó là các thiết bị hồ trợ máy quay giúp tạo nên các góc hình ảnh phản ảnh linh động, bao quát, không bị rung lắc sát thực nội dung mang đến người xem. Thiết bị hỗ trợ đó được đề cập đến trong bài báo này là hệ thống điều khiển camera di chuyển trên đường ray. Hệ thống này trước đây được áp dụng là một hệ thống thô so với hai nguời điều khiển thủ công và chiếm không gian lớn trong phạm vi trường quay và hoạt động rất bị động. Nghiên cứu này nhằm muc tiêu cải tiến với hệ thống điè̀u khiển giúp camera chuyển động đúng ý mong muốn và đặc biệt là hoạt động ổn định, hình ảnh không bị rung lắc khi các động tác pan, tilt camera. Chức năng điều khiển tư động hoạt động ổn định của hệ thống được ưng dụng bằng bộ điều khiển PID (proportional integral derivative). Các kết quả mô phỏng trên $M A T L A B{ }^{\circledR}$ được trình bày và kêt quả thử nghiệm trên mô hình thực tế cho hệ thống này đáp ứng được muc tiêu đặt ra. 


\section{GIỚI THIẸU}

Hầu hết các phim trường đài truyền hình địa phương hiện nay áp dụng hệ thống điều khiển camera thô sơ, với hai người kiểm soát thủ công khả năng di chuyển và điều chỉnh góc quay camera (Hình 1). Hệ thống này chiếm không gian lớn trong phạm vi trường quay và hoạt động kém hiệu quả.

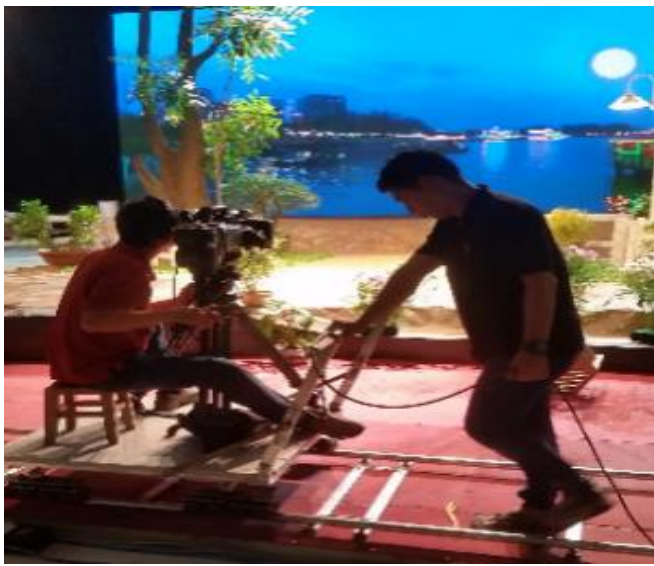

Hình 1. (a) Hệ thống điều khiển camera trên ray thô sơ (b) Trường quay có khán giả
Giá thành nhập khẩu một hệ robot điều khiển camera hiện nay là rất cao. Để có thể tự chủ chế tạo robot điều khiển camera di chuyển trên ray, đòi hỏi các tiêu chí kỹ thuật sau: kích thước nhỏ gọn; chiều cao camera cần đạt khoảng $1,8 \mathrm{~m}$; hệ thống có bốn bánh xe giúp robot di chuyển qua lại trong phạm vi độ dài đường ray ngoài sân khấu; các bánh xe cần có rảnh để không bị rơi khỏi ray và có bộ cảm biến số vòng quay (encoder) để xác định vị trí; bộ phận lắp camera với hai khớp quay: quay ngang (pan) và quay thẳng đứng (tilt) (Lê Văn Chung, 2014; Hiroyuki et al., 2012) với góc pan có thể quay 0$360^{\circ}$ và góc tilt có thể quay $0-100^{\circ}$ tương tự như Hình 2. Ngoài ra robot cũng cần có các chức năng điều khiển khác như: điều khiển phóng to, thu nhỏ khung hình (zoom), điều khiển độ nét của ảnh (focus), và điều khiển khẩu độ không phụ thuộc vào quá trình điều khiển chuyển động của hệ thống.

So với hệ thống điều khiển thủ công thô sơ Hình 1(a), robot điều khiển camera sẽ giúp cải thiện điều kiện không gian trường quay, dễ dàng lắp đặt, cơ động và không ảnh hưởng nhiều đến góc quan sát của khán giả khi trường quay có khán giả ngồi bên dưới Hình 1(b).

Phương pháp điều khiển PID (proportional integral derivative) sẽ được áp dụng cho hệ thống này để khắc phục được các trường hợp rung lắc, dao động và kết quả mô phỏng và thực nghiệm sẽ được trình bày để kiểm chứng kết quả. Bộ điều khiển PID cho hệ thống kiểm soát camera di chuyển trên ray được mô phỏng trước trên một mô hình phi tuyến, nhằm chọn lựa ác thông số $K_{p}, K_{i}, K_{d}$ trước khi đưa vào thực nghiệm. Tính ổn định của hệ thống sẽ được khảo sát thông qua thực nghiệm.

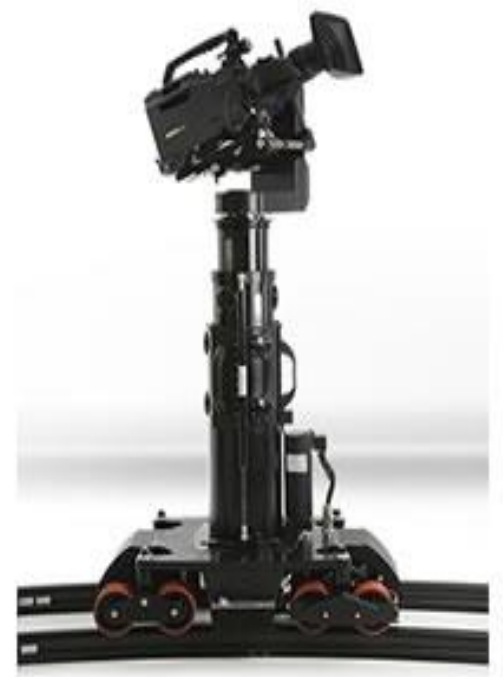

\section{Hình 2. Hệ thống điều khiển camera di chuyển trên đường Ray}

Bài báo được kết cấu thành 5 phần. Động lực học hệ thống được mô tả trong Phần 2; Trong Phần 3 , bộ điều khiển PID được thiết lập để điều khiển đối tượng ổn dịnh; Phần 4 thảo luận kết quả mô phỏng và kết quả so sánh của hệ thống với tín hiệu thực tế; Cuối cùng, kết luận được thảo luận trong Phần 5 và một số hình ảnh minh họa cho mô hình được thiết kế áp dụng trong thực tế trường quay Trung tâm truyền hình Việt Nam Khu vực Nam Bộ - Đài Truyền hình Việt Nam 


\section{MÔ HÌNH TOÁN HỌC CỦA HỆ THỐNG}

Trong phần này, robot điều khiển camera được mô hình hóa tập trung vào động lực học gây ra dao động theo chiều dọc đường ray, và bỏ qua ảnh hưởng của thành phần lắc ngang, do ray được thiết kế dạng đôi. Quá trình nâng cao hạ thấp với biên độ và thời gian nhỏ nên xem như không thay đổi lớn và

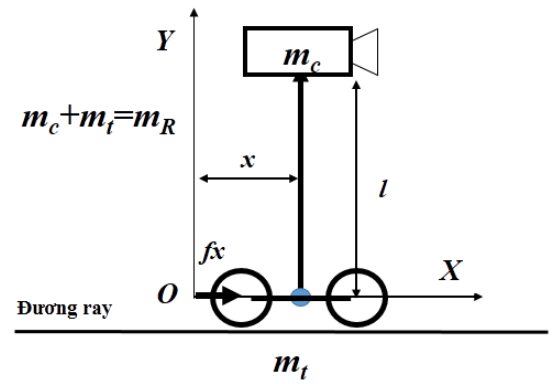

không ảnh hưởng nhiều đến quá trình di chuyển hệ thống (Romano et al., 2003, 2005).

Mô hình động lực học (Nguyễn Ngô Phong \& Ngô Quang Hiếu, 2014; Nidhil Wilfred et al., 2014) của hệ thống điều khiển camera di chuyển trên ray được mô hình hóa vật lý như Hình 3.

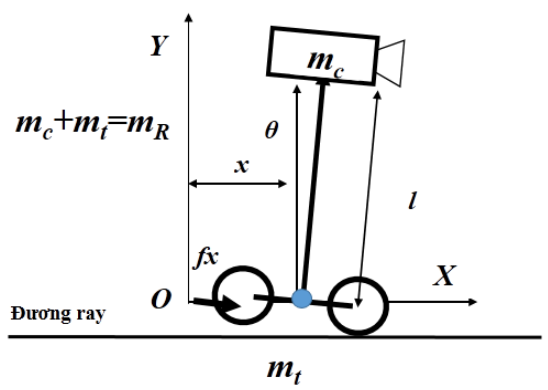

\section{Hình 3. Mô hình hệ thống trên hệ trục tọa độ OXY}

Trong đó:

- $\quad \mathrm{x}$ là vị trí dọc theo trục $\mathrm{X}$ so với điểm khởi động. vị trí của camera và vị trí của bánh xe được xác định $x=x_{t}=x_{c}+l \sin \theta=x_{c}+l(1-\cos \theta)$ Do đó vận tốc camera $\dot{x}=\dot{x}_{c}+l \dot{\theta} \cos \theta \neq \dot{x}_{c}$

- $\theta$ là góc dao động do lức quán tính của quá trình khởi động, tăng tốc, giảm tốc cũng như khi dừng lại của hệ thống so với trục thẳng đứng

- $m_{t}$ và $m_{c}$ khối lượng của bộ phận di chuyển và khối lượng camera

- $l$ là độ cao của camera tính từ tâm camera đến trục bánh xe chuyển,

- $f$ là lực điều khiển tác động đến hệ thống di

- $g$ là gia tốc trọng trường.

Trong quá trình vận hành chiều dài $l$ không đổi. Các khâu ma sát trong chuyển động bánh xe không ảnh hưởng lớn đến độ dao dộng, nên được đơn giản hóa.

Dựa vào chuyển động của xe và tải trọng, động năng $K$ của hệ thống được tính như sau:

$$
K=\frac{1}{2} m_{t} \dot{x}^{2}+\frac{1}{2} m_{c} \dot{x}^{2}=\frac{1}{2}\left(m_{t}+m_{c}\right)(\dot{x})^{2}(1)
$$

Thực tế góc dao động dọc $\theta$ có tồn tại và làm ảnh hưởng đến rung lắc của camera nên thế năng $P$ được tính: $P=m_{c} g l \sin \theta=m_{c} g l(1-\cos \theta)$

Trong đó, $\dot{x}$ là véc-tơ đại diện cho vận tốc của toàn hệ thống, được thể hiện như:

$$
\dot{x}^{2}=\dot{x}_{x}^{2}+\dot{x}_{y}^{2}
$$

Do dao động ngang xấp xỉ bằng không nên $\dot{x}^{2}=$ $\dot{x}_{x}^{2}<=>\dot{x}=\dot{x}_{x}$

Tổng hợp từ (1) và (2)

$$
\begin{gathered}
L=K-P=\frac{1}{2}\left(m_{t}+m_{c}\right)(\dot{x})^{2}-m_{c} g l(1- \\
\cos \theta)=\frac{1}{2} m_{t}(\dot{x})^{2}+\frac{1}{2} m_{c}(\dot{x})^{2}-m_{c} g l(1-\cos \theta)
\end{gathered}
$$

(4)

Lấy đạo hàm $L$ theo biến $x$ ta có:

$\frac{\partial L}{\partial x}=0$

Tiếp theo lấy đạo hàm $L$ theo $\dot{x}$, ta được:

$\frac{\partial L}{\partial \dot{x}}=m_{t} \dot{x}+m_{c} \dot{x}-m_{c} g l \dot{\theta} \sin \theta$

Lấy đạo hàm của (6) theo thời gian $\mathrm{t}$ ta được

$\frac{d}{d t}\left(\frac{\partial L}{\partial \dot{x}}\right)=m_{t} \ddot{x}+m_{c} \ddot{x}-m_{c} g l(\ddot{\theta} \sin \theta+$ $\left.\dot{\theta}^{2} \cos \theta\right)$

Phương trình Lagrange cho $x$ như sau:

$\frac{d}{d t}\left(\frac{\partial L}{\partial \dot{x}}\right)-\frac{\partial L}{\partial x}=\left(m_{c}+m_{t}\right) \ddot{x}-m_{c} g l(\ddot{\theta} \sin \theta+$ $\left.\dot{\theta}^{2} \cos \theta\right)=f$

Phương trình (4) được viết lại:

$L=\frac{1}{2} m_{t} \dot{x}_{t}^{2}+\frac{1}{2} m_{c}(\dot{x}+l \dot{\theta} \cos \theta)^{2}-m_{c} g l+$ $m_{c} g l \cos \theta$

Lấy đạo hàm (9) theo $\theta$ như sau: 


$$
\begin{aligned}
& \frac{\partial L}{\partial \theta}= \\
& m_{c}\left[\begin{array}{l}
(\dot{x}+l \dot{\theta} \cos \theta)(-l \dot{\theta} \sin \theta) \\
+(l \dot{\theta} \sin \theta)(l \dot{\theta} \cos \theta)-m_{c} g l \sin \theta
\end{array}\right]= \\
& -m_{c} l \dot{x} \dot{\theta} \sin \theta-m_{c} g l \sin \theta
\end{aligned}
$$

Lấy đạo hàm (9) theo $\dot{\theta}$ ta được như sau:

$$
\frac{\partial L}{\partial \dot{\theta}}=m_{c}\left[\begin{array}{l}
(\dot{x}+l \dot{\theta} \cos \theta)(l \cos \theta) \\
+(-l \dot{\theta} \sin \theta)(-l \dot{\theta} \sin \theta)
\end{array}\right]=
$$

$m_{c} l \dot{x} \cos \theta+m_{c} l^{2} \dot{\theta}$

Sau đó, ta lấy đạo hàm của (11) theo $t$ ta được

$$
\frac{d}{d t}\left(\frac{\partial L}{\partial \dot{\theta}}\right)=m_{c} l \ddot{x} \cos \theta-m_{c} l \dot{x} \dot{\theta} \sin \theta+m l^{2} \ddot{\theta}
$$

(12)

Phương trình Lagrange đối với $\theta$ như sau:

$$
\frac{d}{d t}\left(\frac{\partial L}{\partial \dot{\theta}}\right)-\frac{\partial L}{\partial \theta}=m_{c} l \ddot{x} \cos \theta+m_{c} l^{2} \ddot{\theta}+
$$

$m_{c} g l \sin \theta=0$

Tổng hợp từ (8) và (13) phương trình động lực học cho hệ thống camera di chuyển trên ray được thể hiện bởi (14):

$$
\left\{\begin{array}{l}
\left(m_{c}+m_{t}\right) \ddot{x}-m_{c} g l\left(\ddot{\theta} \sin \theta+\dot{\theta}^{2} \cos \theta\right)=f \\
\ddot{x} \cos \theta+l \ddot{\theta}+g \sin \theta=0
\end{array}\right.
$$

Phương trình (14) được trình bày lại theo hệ phương trình trạng thái như sau:

$$
\left\{\begin{array}{c}
\dot{x}_{1}=x_{2} \\
\dot{x}_{2}=f_{1}(x)+g_{1}(x) u \\
\dot{x}_{3}=x_{4} \\
\dot{x}_{4}=f_{2}(x)+g_{2}(x) u
\end{array}\right.
$$

Trong đó, $x=\left[\begin{array}{llll}x_{1} & x_{2} & x_{3} & x_{4}\end{array}\right]^{T}, \quad x_{1}=x$, $x_{3}=\theta, x_{2}$ là vận tốc dài, $x_{4}$ là vận tốc góc hệ thống, $u$ là tín hiệu điều khiển, và $f_{i}, g_{i}(i=1,2)$ được biểu diển như sau:

$$
\begin{aligned}
& f_{1}(x)=\frac{m_{t} l x_{4}^{2} \sin x_{3}+m_{c} g \sin x_{3} \cos x_{3}}{m_{t}+m_{c} \sin ^{2} x_{3}} \\
& g_{1}(x)=\frac{1}{m_{t}+m_{c} \sin ^{2} x_{3}}
\end{aligned}
$$

$$
\begin{aligned}
& f_{2}(x)=\frac{\left(m_{t}+m_{c}\right) g \sin x_{3}+m_{c} l x_{4}^{2} \sin x_{3} \cos x_{4}}{\left(m_{t}+m_{c} \sin ^{2} x_{3}\right) l} \\
& g_{2}(x)=\frac{\cos x_{3}}{\left(m_{t}+m_{c} \sin ^{2} x_{3}\right) l}
\end{aligned}
$$

\section{THIẾT KẾ BỘ DIỀU KHIỂN PID}

Bộ điều khiển PID là một cơ chế hồi tiếp vòng kín kinh điển, được sử dụng rộng rãi trong các hệ thống điều khiển công nghiệp. Bộ điều khiển PID sẽ tính toán tín hiệu điều khiển, dựa theo giá trị sai biệt giữa đáp ứng ngõ ra và giá trị đặt mong muốn. Bộ điều khiển sẽ thực hiện giảm tối đa sai số bằng cách điều chỉnh giá trị điều khiển đầu vào (Nguyễn, 2015; Nguyễn, 2017).

Giải thuật tính toán bộ điều khiển PID bao gồm 3 thông số riêng biệt, do đó đôi khi nó còn được gọi là điều khiển ba khâu: các giá trị tỉ lệ, tích phân và đạo hàm, viết tắt là $\mathrm{P}, \mathrm{I}$, và $\mathrm{D}$. Giá trị tỉ lệ xác định tác động của sai số hiện tại, giá trị tích phân xác định tác động của tổng các sai số quá khứ và giá trị vi phân xác định tác động của tốc độ biến đổi sai số. Tổng chập của ba tác động này dùng để điều chỉnh quá trình thông qua một phần tử điều khiển. Nhờ vậy, những giá trị này có thể làm sáng tỏ về quan hệ thời gian: $\mathrm{P}$ phụ thuộc vào sai số hiện tại, I phụ thuộc vào tích lũy các sai số quá khứ và $\mathrm{D}$ dự đoán các sai số tương lai, dựa vào tốc độ thay đổi hiện tại. Đây là một bộ điều khiển phổ dụng, nên phần này không trình bày sâu về bộ điều khiển PID.

Đối với hệ thống này này, bộ điều khiển PID được điều chỉnh theo phương pháp thử-sai cho đến khi hệ thống đạt ổn định, sau đó tiến hành thực nghiệm.

\section{KÊT QUẢ MO PHỎNG VÀ THỰC NGHIẸM}

Sơ đồ mô phỏng robot được xây dựng trên MATLAB bằng khối S-Function như Hình 4. Các thông số của mô hình robot là các thông số thực tế đo đạc, được trình bày trên Bảng 1 . Sau quá trình mô phỏng, thông số của bộ điều khiển PID được xác lập và cài đặt trên board mạch thực tế Hình 5 . Thực nghiệm trên robot thật sẽ thu thập kết quả và so sánh với mô phỏng, giúp việc chọn lựa thông số bộ PID hiệu quả nhất. 


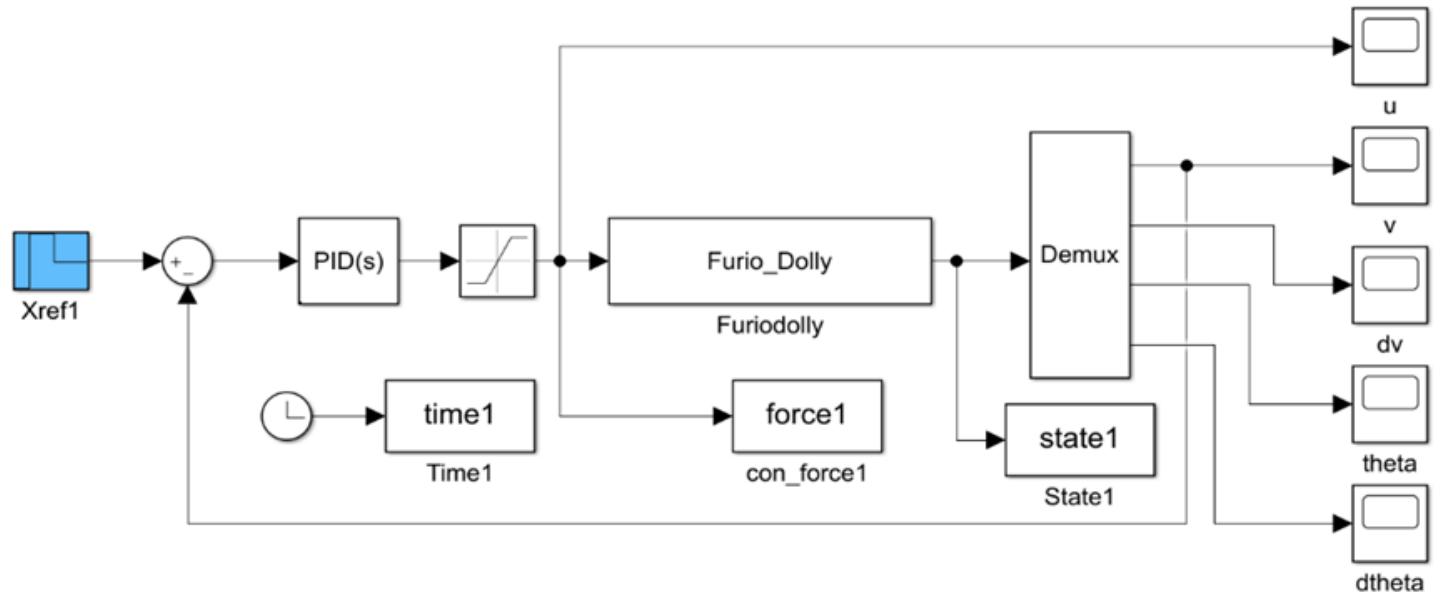

Hình 4. Sơ đồ mô phỏng robot điều khiển Camera trên MATLAB

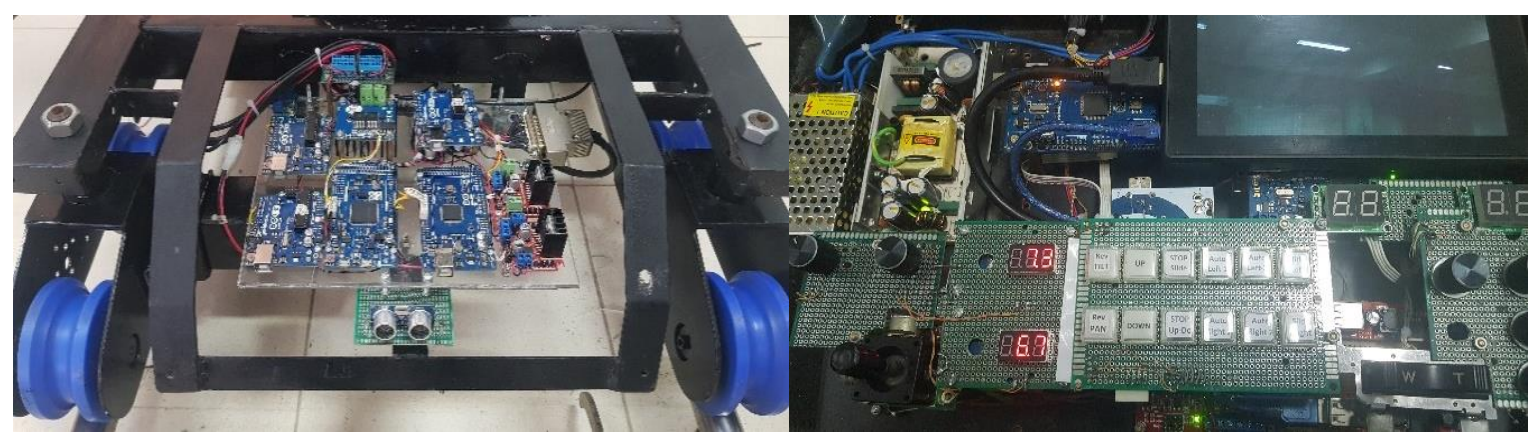

a)- Bên trong bộ phận di chuyển

b)- Bên trong bàn điều khiển

Hình 5. Board mạch điều khiển trên hệ thống thực tế

Bảng 1. Thông số của hệ thống

\begin{tabular}{clc}
\hline STT & Giá trị & Thông số \\
\hline 1 & $m_{c}-$ khối luợng camera $(\mathrm{kg})$ & 3,5 \\
2 & $m_{t}-$ khối luợng dolly $(\mathrm{kg})$ & 20 \\
\hline 3 & l-chiều cao camera $($ mét $)$ & 1,7 \\
\hline
\end{tabular}

Bảng 2. Thông số $K_{p} K_{i} K_{d}$ sau khi thử lựa chọn tốt nhất

\begin{tabular}{clc}
\hline STT & Giá trị & Thông số \\
\hline 1 & $K_{p}$ & 13,8 \\
2 & $K_{i}$ & 0.5 \\
3 & $K_{d}$ & 8.8 \\
\hline
\end{tabular}

Khi hệ thống điều khiển camera nhận được tín hiệu tác động nó sẽ di chuyển, tuy nhiên để vừa di chuyển vừa giữ ổn định camera thì cần có tín hiệu điều khiển từ bộ điều khiển giữ camera di chuyển ổn định. Đáp ứng vận tốc robot thật và kết quả mô phỏng, tương ứng với tín hiệu điều khiển do bộ PID cung cấp (Bảng 2 ) được trình bày trên Hình 6 . Đáp ứng vận tốc thực tế trên Hình 6 tồn tại sai số xác lập, do khâu $\mathrm{K}_{\mathrm{I}}$ chưa được chọn lựa tốt. Tuy nhiên, sai số này rất nhỏ và kết quả thực tế cho thấy robot di chuyển đạt yêu cầu tác nghiệp, nên kết quả có thể chấp nhận được. 

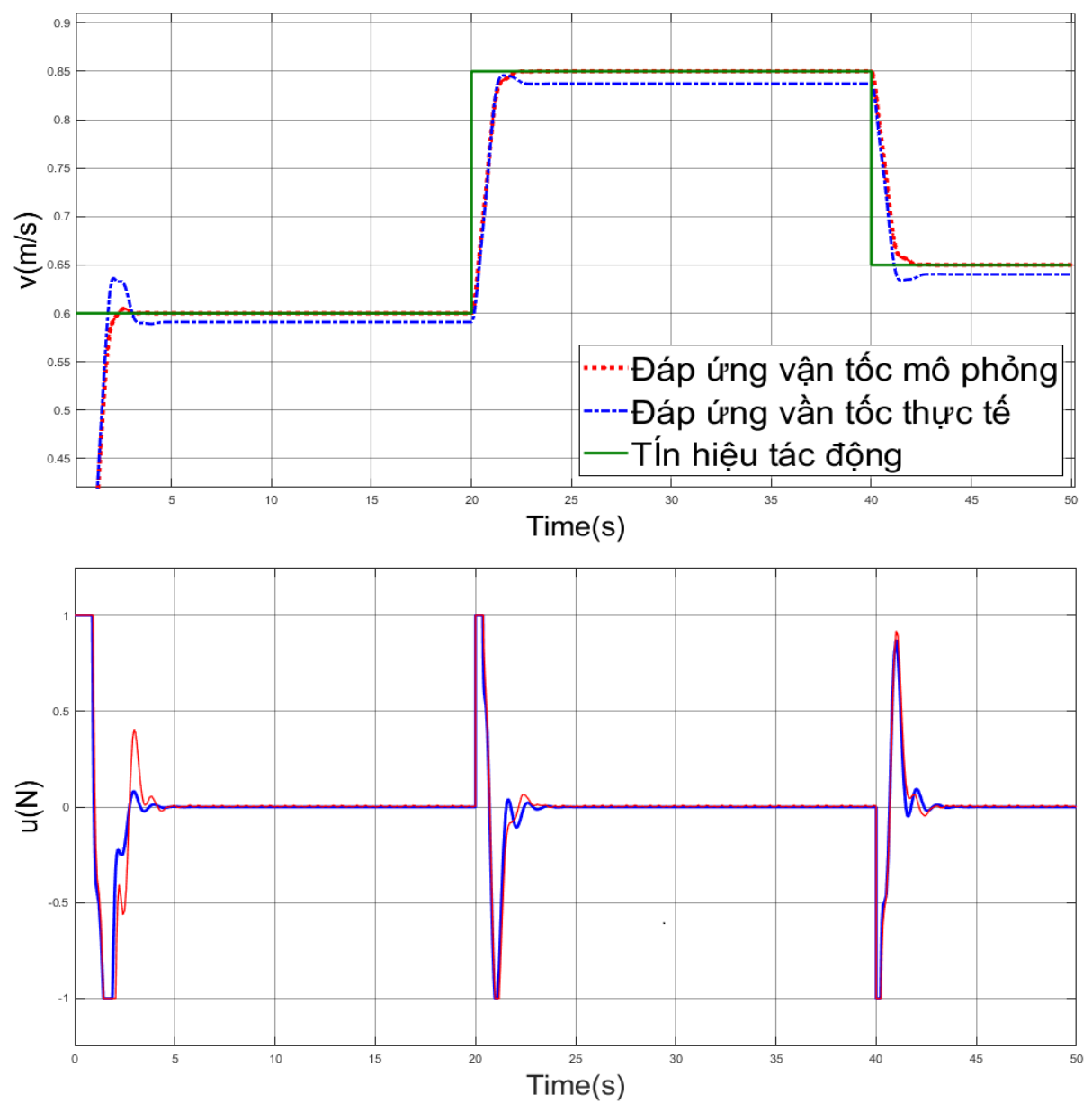

Hình 6. Đáp ứng vận tốc và tín hiệu điều khiển

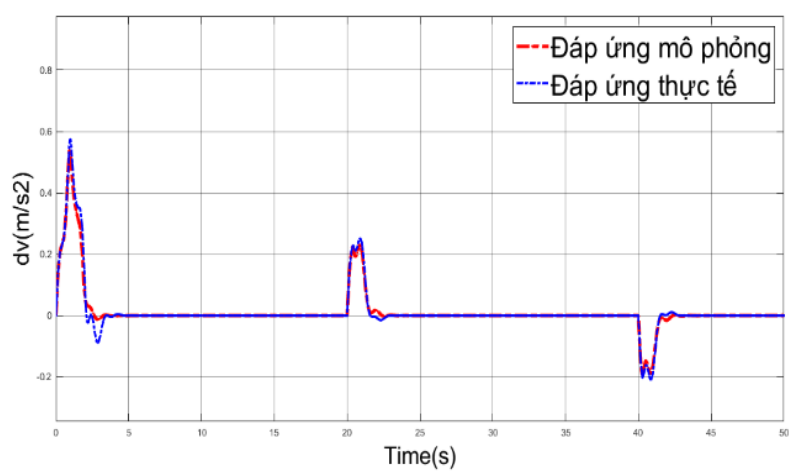

(a)

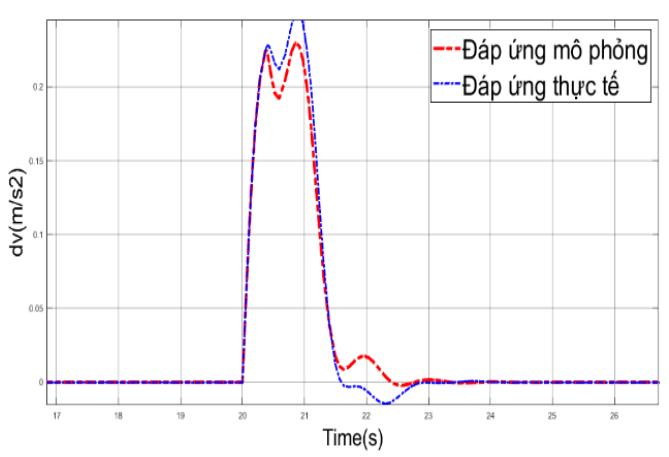

(b)

Hình 7. Đáp ứng gia tốc của hệ thống Robot khi di chuyển

\section{(Hình b là phóng to của Hình a tại các khoản thời gian thay đổi)}

Đáp ứng gia tốc của robot được trình bày trên Hình 7. Theo kết quả này, ở mỗi thời điểm chuyển trạng thái thì vận tốc robot thay đổi do đó gia tốc cũng có thay đổi, nhưng chỉ hơn 01 giây là tiến về không $(0)$ do bộ điều khiển đã kiểm soát robot với vận tốc mới đã xác lập ổn định. 


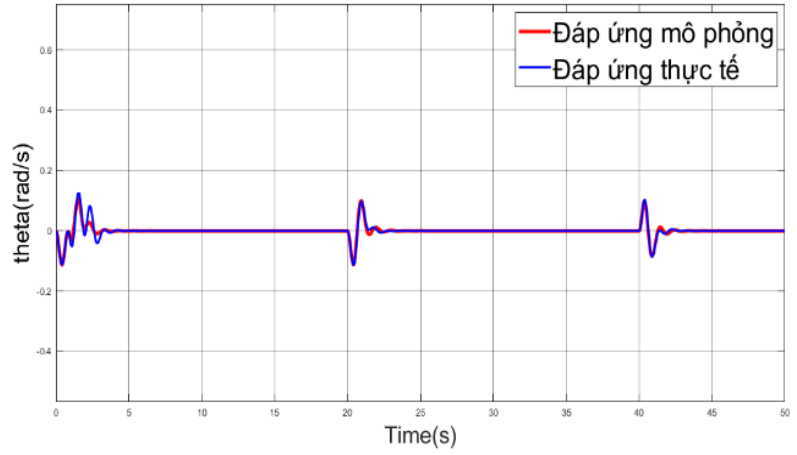

(a)

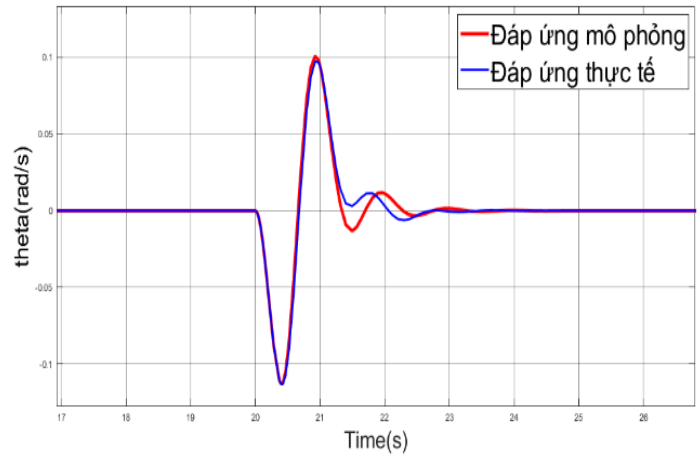

(b)

Hình 8. Đáp ứng ngõ ra góc dao động dọc $\theta$ khi Robot di chuyển

(Hình (b) là phóng to Hình (a) tại thời điểm thay đổi)

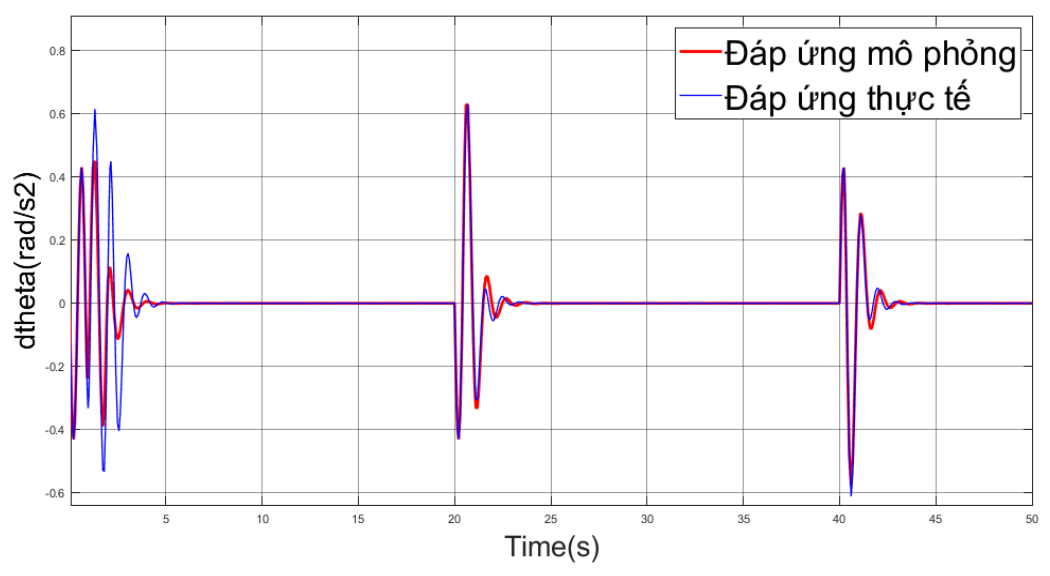

Hình 9. Đáp ứng ngõ ra vận tốc góc của Robot khi di chuyển

Để kiểm chứng tính ổn định, nghiên cứu tiến hành khảo sát đáp ứng góc dao động và vận tốc góc trên Hình 8 và Hình 9. Quan sát Hình 8 , tương ứng tại các thời điểm chuyển trạng thái sẽ xuất hiện tín hiệu điều khiển cho thấy lực tác động tăng lên nên hệ thống di chuyển nhanh lên. Góc dao động $\theta$ ở Hình 9 có biến đổi mỗi khi tăng hoặc giảm tốc độ hệ thống.

\section{THẢO LUẬn VÀ ĐÁNH GIÁ KẾT QUẢ ĐẠT ĐƯợC}

Để thấy được tính hiệu quả và sự hoạt động ổn định của hệ thống, ta cho hệ thống hoạt động trong thời gian 50s, thời gian đủ để đánh giá được tình ổn định lâu dài của hệ thống.

Dựa vào các kết quả mô phỏng ta thấy được:

Khi tác động vào hệ thống một lực $f$ thì hệ thống sẽ chuyển động với vận tốc $v$ trung bình tù $0.6 \mathrm{~m} / \mathrm{s}$ đến $1 \mathrm{~m} / \mathrm{s}$ (Hình 6) (vận tốc phù hợp với yêu cầu thực tế), lúc này bộ điều khiển PID điều khiển hệ thống di chuyển đến tốc độ xác định đồng thời tại các thời điểm thay đổi sẽ xuất tín hiệu điều khiển để giữ hệ thống ổn định với góc dao động $\boldsymbol{\theta}$ (Hình 8) có biên độ dao động nhỏ (không đáng kể), và nhanh tiến về không trong hơn 01 giây. Sự thay đổi và biến mất nhanh của gia tốc (Hình 7) và góc dao động $\boldsymbol{\theta}$ cho thấy hệ thống hoạt động ổn định.

Đáp ứng của bộ điều khiển PID (đáp ứng đường màu đỏ) với hệ thống điều khiển Camera di chuyển trên đường Ray được thiết lập trên Matlab đã cho thấy tác dụng ưu việc của nó trong việc giữ ổn camera khi di chuyển.

Đối với đáp ứng bộ điều khiển PID cho thực tế hệ thống (đáp ứng đường màu xanh) thì cũng cho kết quả rất tốt, tại các điểm chuyển trạng thái có xuất hiện thay đổi về vận tốc (độ vọt lố cao nhất là $8 \%$ ) gia tốc và góc dao động nhanh tiến về không (chỉ hơn 01 giây). Qua quan sát hình ảnh ghi hình thì sử dụng được các thời điểm đó. Trong thực tế về nguyên tắc sử dụng hình ảnh của robot di chuyển trên Ray này là không cần phải tác động thay đổi tốc độ quá nhanh, nó còn liên quan đến các thao tác 
zoom hình ảnh nên độ rung lắc sẽ không bị ảnh hưởng khi hoạt động

Nghiên cứu tiếp theo sẽ được đề xuất là phương pháp kết hợp Fuzzy-PID (Umesh and Rakesh, 2013) hoặc điều khiển trượt (SMC) sẽ được thực hiện để khắc phục tốt hơn các thành phần phi tuyến khác và triệt tiêu toàn bộ góc dao động $\theta$ (dao động dọc)

Mô hình hệ thống điều khiển Camera di chuyển trên đường Ray đang sử dụng bộ điều khiển PID được áp dụng cho thiết kế robot thực tế

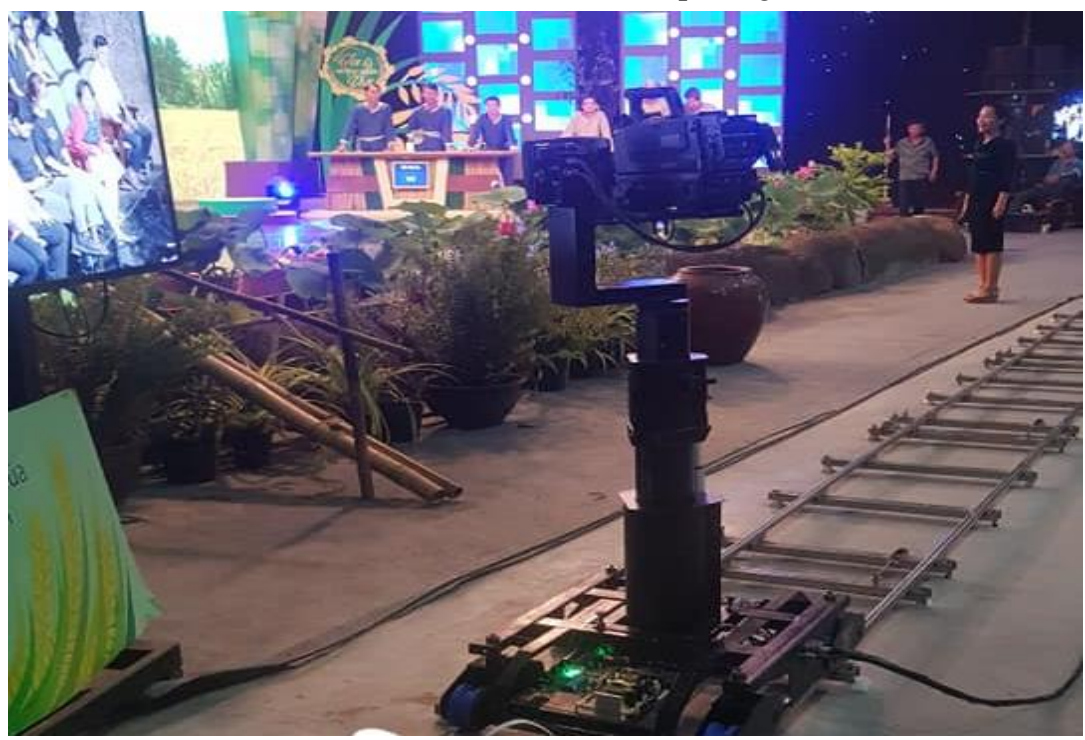

Hình 10. Hệ thống Robot được vận hành thử nghiệm tại trường quay Trung tâm THVN Khu vụcc Nam Bộ

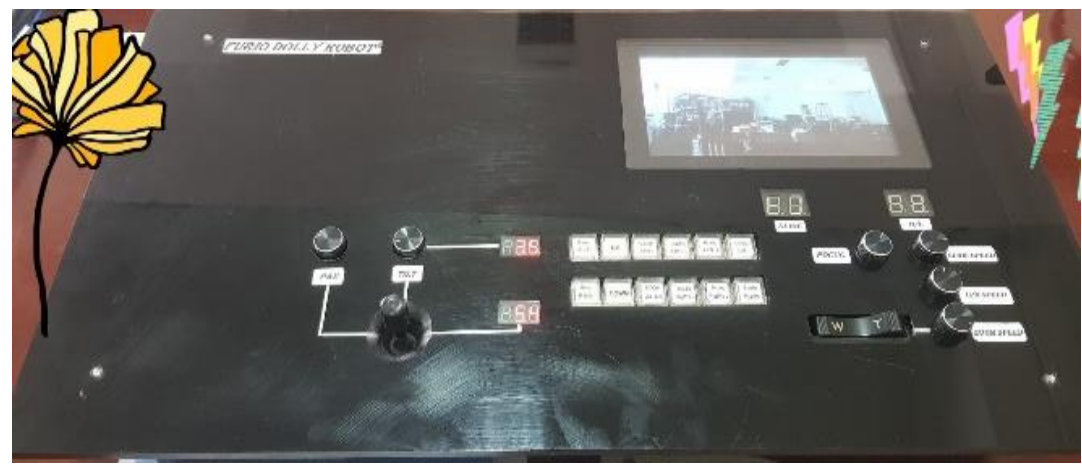

Hình 11. Bàn điều khiển hệ thống

\section{KẾT LUẬN}

Bài báo đã trình bày đầy đủ về phương pháp đề xuất điều khiển hệ thống và trình bày các kết quả mô phỏng đã đạt được mục tiêu đặt ra. Hệ thống đã được thiết kế và chạy thực tế tại trường quay (Nguyễn, 2020), các kết quả mô phỏng cho thấy hệ thống hoạt động đảm bảo tính ổn định theo mong muốn. Mục tiêu phát triển tiếp theo của hệ thống là sử dụng các phương pháp điều khiển khác để có bộ điều khiển hệ thống đáp ứng tốt hơn.

Bên cạnh những thành công đã đạt được thì nghiên cứu này vẫn còn tồn : chưa khảo sát độ thay đổi nhanh chiều cao của hệ thống, khảo sát một mô hình có tải trọng nhẹ hơn nhiều lần so với hệ thống này,...

Trong các nghiên cứu tương lai sẽ ứng dụng phương pháp điều khiển trượt (Bartolini et al., 2000) để xử lý các thành phần phi tuyến bấc định, phương pháp xử lý ảnh cũng sẽ được đề xuất vào việc phát hiện đối tượng và di chuyển bám theo đối tượng, ứng dụng trong các chương trình truyền hình về thể thao sẽ được nghiên cứu và phát triển ở các bài sau.

\section{TÀI LIỆ THAM KHẢO}

Bartolini, G., Ferrara, A., Usai, E and Utkin, V. I. (2000). On Multi-input second order sliding 
mode control”, IEEE Trans. Aut. Contr., 45(9), 1711-1717.

Bartolini, G., A. Pisano, A. \& Usai, E. (2000). First and Second Derivative Estimation by Sliding Mode Technique, Journal of Signal Processing, 4(2), 167-176.

Ukida, H., Terama, Y., \& Ohnishi, H. (2012). Object Tracking System by Adaptive Pan-tilt-zoom Cameras and Arm Robot. Proceeding of SICE Annual Conference, 1920 - 1925.

Wilfred, K. J., Sreeraj, S., Vijay, B., \& Bagyaveereswaran, V. (2014). Container Crane Control using Sliding Mode Control. International Journal of Engineering Research \& Technology, 3(6), 2278-0181.

Lê Văn Chung. (2014). Phát triển hệ pan-tilt nhiều camera bám mục tiêu di động. Tạp chí Khoa hoc và Công nghệ Đại học Công nghệ thông tin và Truyền thông - Đại học Thái Nguyên, 116(2), 41- 46.

Nguyễn Hoàng Vương. (2020). Hệ thống robot điều khiển camera VTV ( truy cập ngày 26/10/2020). https://www.youtube.com/watch?v=FlldrjWu23E

Nguyễn Ngô Phong \& Ngô Quang Hiếu. (2014). Sliding mode control of two dimensional container cranes. Hội nghị toàn quốc lần thư 7 về Co điện tư (VCM-2014), 394-399.

Nguyễn Thanh Tần. (2017). Thiết kế mô hình cần bằng con lắc ngược. Báo cáo Đề tài Nghiên cúu Khoa hoc - Truò̀ng Đại hoc Trà Vinh. https://khcn.tvu.edu.vn/files/project/bao_cao_ton g_ket_file/529/N.T.TAN\%20$\% 20$ Mo\%20hinh\%20can\%20bang\%20con\%20la c\%20nguoc.pdf

Nguyễn Văn Sơn. (2015). Xây dựng mô hình con lắc ngược dạng quay trên Simulink Matlab. Tạp chí Đại học Thủ Dầu Một, 6(25), 48-56

Romano, V. F., \& da Trindade, E. C. (2005). Dynamic modeling of a high speed trolley for TV transmission. In ABCM Symposium Series in Mechatronics (Vol. 2, 242-249).

Romano, V. F., Ferreira, C. V., \& Ferreira, C. G. (2003). Mechatronical design of a telerobotic trolley for the TV transmission. IFAC Proceedings Volumes, 36(17), 85-90.

Bansal, U. K., \& Narvey, R. (2013). Speed control of DC motor using fuzzy PID controller. Advance in Electronic and Electric Engineering, 3(9), 1209-1220. 